\title{
Chapter 1 \\ Emerging and Transboundary Animal Viral Diseases: Perspectives and Preparedness
}

\author{
Mahendra Pal Yadav, Raj Kumar Singh, and Yashpal Singh Malik
}

\begin{abstract}
The epidemics and pandemics of a few infectious diseases during the past couple of decades have accentuated the significance of emerging infectious diseases (EIDs) due to their influence on public health. Although Asia region has been identified as the epicentre of many EIDs and upcoming infections, several new pathogens have also emerged in the past in other parts of the world. Furthermore, the emergence of new viral diseases/infections, such as Rift Valley fever, West Nile fever, SARS coronavirus, Hendra virus, avian influenza A (H5N1), Nipah virus, Zika virus and swine influenza A (H1N1) virus, from time to time is a glaring example threatening adversely both animal and public health globally. Infectious diseases are dynamic and concerning due to their epidemiology and aetiological agents, which is manifested within a host, pathogen and environment continuum involving domestic animals, wildlife and human populations. The complex relationship among host populations and other environmental factors creates conditions for the emergence of diseases. The factors driving the emergence of different emerging infectious disease (EID) interfaces include global travel, urbanisation and biomedical manipulations for human EIDs; agricultural intensification for domestic animal EIDs; translocation for wildlife EIDs; human encroachment, ex situ contact and ecological manipulation for wildlife-human EIDs; encroachment, new introductions and 'spill-over' and 'spill-back'; and technology and industry for domestic animal-human EIDs. The concepts of sanitary and phytosanitary (SPS) measures and biosecurity have gained recognition globally in almost all the realms of human activities, including livestock health and production management. This chapter provides the experience gained in the control and management of a few important
\end{abstract}

\author{
M. P. Yadav \\ Sardar Vallabh Bhai Patel University of Agriculture and Technology, \\ Meerut, Uttar Pradesh, India \\ ICAR-Indian Veterinary Research Institute, Izatnagar, Uttar Pradesh, India \\ R. K. Singh · Y. S. Malik ( $ه)$ \\ ICAR-Indian Veterinary Research Institute, Izatnagar, Uttar Pradesh, India
}


TADs and EIDs along with the successes, constraints, limitations and future research needs for developing better control approaches.

Keywords Transboundary animal disease $\cdot$ Infectious animal diseases $\cdot$ Emerging diseases - Biosecurity and biosafety - Animal virus diseases $\cdot$ Disease management

\subsection{Preamble}

A 'disease' may be defined as a condition of the living human, animal or plant body or one of its parts that impairs normal physiological functioning and is typically manifested by clinical symptoms and signs. Animal and human diseases are classified in several ways depending on the criteria, such as infectious and non-infectious diseases, contagious and non-contagious diseases, zoonotic and non-zoonotic diseases, and acute and chronic diseases. The infectious diseases are caused by virus, bacteria, mycoplasma, fungi or rickettsia depending on the nature of the etiological agent involved. Among the infectious diseases, viral diseases are most devastating and difficult to control as they usually spread very fast and unlike other infectious diseases have no cost-effective or safe antiviral antibiotics/drugs for the treatment. A new disease occurring in an area/host population spread further and get established in want of awareness, lack of diagnostic facility, expertise or other factors is termed as 'emerging disease'. While disease of past, re-appearing in an area having a susceptible host population(s), is termed as 're-emerging disease' (Lederberg et al. 1992; Daszak et al. 2000). Example of the latter is the re-emergence of human tuberculosis in many countries which earlier had negative status as a result of judicious control measures adopted; it re-appeared subsequently due to the spread of immunosuppressive human AID's virus, namely human immunodeficiency virus (HIV). Introduction of susceptible animal populations/breeds from abroad for crossbreeding to upgrade the local breeds in India and other developing countries has resulted in the re-emergence of protozoon parasitic infections, such as theileria, babesia and anaplasma in the imported stock or their crossbreeds. The disease which is introduced from abroad into a country is termed as 'exotic'.

The infectious diseases are dynamic concerning their epidemiology and etiological agents which is manifested within a host, pathogen and environment continuum involving domestic animals, wildlife and human populations. The complex relationship between these host populations and other environmental factors creates conditions for disease emergence. The spill-over of the new emerging disease may be from 'domestic animals to wild life', 'wildlife to humans' or 'domestic animal to human' or in all categories. The factors driving the emergence of different EID interfaces include global travel, urbanisation and biomedical manipulations for human EIDs; agricultural intensification for domestic animal EIDs; translocation for wildlife EIDs; human encroachment, ex situ contact and ecological manipulation 
for wildlife-human EIDs; encroachment, new introductions and 'spill-over' and 'spill-back'; and technology and industry for domestic animal-human EIDs.

\subsection{Emerging Diseases}

The term 'emerging disease' is used to refer to changes in the disease dynamics in the population. Emerging infectious diseases (EIDs) are those which have moved recently into a new host or have enhanced incidences or geographic range or are caused by evolving pathogens (Lederberg et al. 1992; Daszak et al. 2000). This general definition covers a range of infectious diseases of man and animals which pose a significant threat to both medical and veterinary public health. Among the OIE-listed diseases of viral aetiology, major changes have been experienced in the occurrence of rinderpest, peste-des-petits ruminants (PPR), foot-and-mouth disease (FMD), African swine fever (ASF), lumpy skin disease and Rift Valley fever (RVF). Of these, rinderpest presents a success story from the 1990s to 2011 as a result of FAO, OIE, EU and IAEA (International Atomic Energy Agency) guided and coordinated programmes including the Pan African Rinderpest Campaign (PARC), NPRE and NREP in India (Yadav 2011), Global Rinderpest Eradication Program (GREP) of the FAO and other national governments where the disease was endemic. These exemplary efforts led to the historic declaration of global rinderpest eradication by the FAO on June 28, 2011.

\subsection{Transboundary Diseases}

The terms 'exotic disease' and transboundary animal diseases (TADs) are often used interchangeably. Though all transboundary diseases are of exotic origin, all exotic diseases are not included in TAD listing. Many EIDs are also transboundary diseases. The TADs are defined as highly contagious and transmissible epidemic diseases of livestock which have the capability for rapid spread to new areas and regions regardless of national borders and have serious socio-economic and public health consequences. Nearly all diseases affect livestock, poultry, fishes and other animals and adversely impact the quality and quantity of food and other products, such as hides and skins, bones, fibres, wool and animal draft power for tilling, transport and traction. The reduction in animal production, productivity and profitability due to TADs affect the human livelihood. In the present scenario of fast-increasing globalisation, TADs represent a serious threat to the economy and welfare of the public and affected nations as they drastically reduce production and productivity; disrupt trade and travel and local and national economies; and also threaten human health through inferior food quality and zoonotic diseases/infections. As such, consequences of TADs could have a significant detrimental effect on the economy and public health of not only the affected nations but also the whole of the world. 


\subsubsection{Source of the Pathogens of EIDs and TADs}

Possibly the infectious agents which cause emerging and transboundary diseases are already present in the environment and get the opportunity to cause disease under certain altered circumstances. The transmission of the infectious agent could occur between animal and human; between wildlife, human and domestic animals; or between wildlife, domestic animal(s) and human. However, the main source for maintenance and transmission of the infectious agents in nature is determined by the zoonotic pool and spill-over and spill-back mechanisms.

\subsubsection{Transboundary Diseases as Potential Threats}

TADs have become of great concern due to the risk for national security on account of their economic significance, zoonotic nature and ever-growing threat of newer TADs in future. Among the TADs having zoonotic manifestations, a number of infectious diseases, such as highly pathogenic avian influenza (HPAI), BSE (Mad cow disease caused by prion), West Nile fever, Rift Valley fever, SARS coronavirus, Hendra virus, Nipah virus, Ebola virus, Zika virus and $\mathrm{CCHF}$, to name a few, adversely affecting animal and human health have been in the news in recent times (Malik and Dhama 2015; Munjal et al. 2017; Singh et al. 2017, 2019). The direct and indirect costs due to the FMD outbreak in the UK in 2001 were assessed to be over US\$9 billion. Over 150 million chicken died or were destroyed in Southeast Asia in 2004 to control HPAI (H5N1). The Netherlands suffered an economic loss of $\$ 2.5$ billion due to classical swine fever in 1997-1998. As per the estimates of FAO nearly one-third of the world meat trade was facing import bans on account of BSE, HPAI and other animal diseases. There is evidence to suggest that threats from TADs have increased over the years. The risk of animal disease outbreaks is likely to further grow in future as the higher incomes of people in developing countries will generate more demand for animal protein and products (milk, meat, egg, chicken and fish). The number of animals raised for meat is growing rapidly. During 1990 s poultry production in East Asia has increased by about $12 \%$ per year to double every 5-6 years. Similar to TADs, new human viral diseases have emerged like Ebola, SARS, Zika, CCHF, Nipah and BSE as well as there is the emergence of new antigenic forms or new biotypes of the existing infectious diseases, such as a hypervirulent strain of IBD in poultry in Europe and highly virulent strain of Newcastle disease in the USA (Riemenschneider 2005; Singh et al. 2017). Vector-borne pathogens, namely, bluetongue, African horse sickness, Rift Valley fever and West Nile fever, have the potential to spread in epidemic forms. Riemenschneider (2005) has deliberated over several issues relevant in the control of TADs as proposed in the Institute of Medicine (IOM) Report (Anonymous 2003). Some of the points which could be responsible for the increased threat of TADs are briefly discussed below. 


\subsubsection{Globalisation and Trade}

In the present-day world, higher quantitative levels of animal origin foods, as well as faster trade, new trade routes and air travel, have led to higher risks for contracting new infections and diseases. As it is now possible to reach any part of the world within $24 \mathrm{~h}$ which is less than the incubation period of most of the infectious diseases, animals or people carrying the infectious agents go undetected in want of clinical disease/symptoms. Fresh commodities vis-à-vis processed foods that have witnessed an increased trade are more likely to carry the pathogens to distant parts of the world-countries and continents.

\subsubsection{Intensive Animal Production Systems to Meet the Rising Demand for Animal Protein}

Recent decades are witnessing higher demands for animal protein and other nutrients through meat and meat products, milk and milk products, eggs, and fish and fish products as a result of rising incomes in the developing countries and elsewhere which leads to the intensification of production systems and overcrowding of animals. This increased production is often required in peri-urban areas, having large human populations, under suboptimal husbandry practices. In such high-production areas, disease outbreaks affect a greater number of animals at a faster rate and speed, leading to heavy economic losses. Drastic control measures are taken, such as the slaughter of infected and in-contact animals followed by burning or burial is not acceptable to the society at large. For example, the mass slaughter of pigs in the Netherlands in 1997-1998 for the control of CSF virus led to objection from the non-farm population which might influence the application of the stamping-out policy as a disease control approach in future.

\subsubsection{Impact of Changes in Forest Ecology}

Exposure of the domestic animals to forest niches due to deforestation and transformation of tropical rainforests for livestock grazing exposes the domestic livestock to a completely new range of pathogens and vectors which previously circulated in wildlife reservoir niches only. With the domestic livestock being fully susceptible and naïve to these infectious agents, the disease spreads more rapidly and severely in want of lack of diagnostic tests and vaccines against these new pathogens resulting in heavy morbidity, mortality, trade restrictions and economic losses. 


\subsubsection{Influences of Increased Conflicts and Unrest}

Nowadays many countries face prolonged civil unrests besides inter- and intracountry conflicts, which may lead to enhanced threat of TADs. Civil disorders are known to disrupt enforcement of quarantine and other control measures due to refugee and army deployments/movements. Breakdown in the institutional support for quarantine and difficulty in gaining access to border area due to landmines make disease surveillance more difficult. Inflows of more food aids for such areas also pose additional risks as the food items may have contaminants.

\subsubsection{Effect of Climate Change, Global Warming and Microbial Evolution}

Climate change and global warming seem to be altering rainfall and weather patterns. Rising temperatures in the northern hemisphere are likely to shift the distribution of insect vectors of bluetongue, African horse sickness, Rift Valley fever and similar vector-borne diseases. The bluetongue virus (BTV) having 27 serotypes occurs in many parts of the world. However, until recently it was never reported from Europe. The sudden incursions of some serotypes into Spain, Italy, Greece, Portugal and the Balkan countries since 1998, followed by Germany, and the recent incursion of BTV serotype 8 in several farms in the Netherlands, Germany and Belgium since August 2006 as well as serotype 1 are also believed to be due to climate change as European weather has become hotter in recent decades. The BTV serotype 8 revealed that this serotype is closest to the Nigerian strain. The incursion is believed to have been caused by the importation of an infected zoo animal or an infected midge. An upsurge of Rift Valley fever was observed in East and West Africa due to climatic changes.

\subsubsection{TADs as a Serious Threat to National Security}

Many factors discussed above make the TADS as a serious threat to national and international security. The developing countries are usually the worst sufferers. Among other factors, veterinary public health services in developing countries are usually much behind than the medical public health services. Moreover, unlike human disease reporting, animal disease reporting systems are usually based on passive reporting rather than active disease surveillance. A few other factors are also responsible for greater threat due to TADs, namely (1) lack of awareness of the farmers about the high-threat epizootic animal diseases; (2) lack of diagnostic facilities for exotic diseases, and under-reporting of animal diseases like HPAI due to the fear of loss of internal and export market till the country gets infection-free status as per OIE-laid-down criteria; and (3) poor and faulty compensation schemes. 


\subsection{Threat of Bioterror/Biowarfare}

In the technological advances made in today's world, there is always a real risk of deliberate misuse of certain infectious agents/pathogens by terrorists as a means of biowarfare between nations to harm the people and/or livestock, poultry and other animals. Potential for pathogenic disease agents not reported previously in a country and being misused or mishandled for bioterrorism is likely to threaten the ecosystem on a large scale. Even new pathogens can be engineered as novel infectious agents. The animal diseases could even be a greater threat than human diseases as these may result in significant economic disruptions, besides causing food poisoning and deterioration, and zoonotic diseases in human beings. As animal diseases get less priority than human infections/diseases in undertaking immediate disease control measures, the threat scenario with the use of animal pathogens for bioterrorism or biowarfare will have many serious consequences. Some of the viruses having significant bioterror potential for humans and or animals include HPAI (H5N1), monkeypox virus, FMD virus, yellow fever virus, Spanish flu virus, poliovirus, AIDS virus (HIV), measles virus, Hendra virus, Nipah virus, SARS coronavirus, BSE prion agent and unknown agent(s) created through biotechnology and gene editing.

\subsection{Handling of New Viral Disease Outbreaks}

When an exotic viral disease strikes a country for the first time, it may initially affect one animal, few animals or a large number of animals. The strategy to be adopted for containing the outbreak will depend on the nature of the virus, speed of its spread, role of vectors, risk assessment, communication and management, response time and country legislation on disease control and prevention. Thus, there is a need to develop strategic plans for the prevention and control of exotic and TAD on a case-to-case basis. Examples of such viral diseases from Indian perspective include African swine fever (ASF), transmissible gastroenteritis (TGE), and swine vesicular disease in pigs, Rift Valley fever, African horse sickness (AHS), West Nile fever, Eastern equine encephalomyelitis (EEE), Western equine encephalomyelitis (WEE), and Venezuelan equine encephalomyelitis (VEE), FMD virus types ' $C$ ', 'SAT I', 'SAT II' and 'SAT III', Nipah virus, Hendra virus, SARS coronavirus, and prion diseases-bovine spongiform encephalopathy (BSE), and scrapie.

\subsection{Biosecurity and Biosafety Measures to Combat Viral Infections}

Institution of appropriate and timely biosecurity measures is an important instrument for the protection and improvement of animal health. Breach in biosecurity due to ignorance and avoidable lapses in the adoption of timely biosecurity and 
biosafety measures in the management of livestock, poultry and fish minimise the risks from infectious diseases including EIDs and TADs. Breach in biosecurity in livestock management is often an important reason for the high incidence of zoonotic and other infectious diseases of animals. This is more so in case of the viral diseases of livestock and poultry. Closer contact between wildlife, animals and humans and rearing of livestock and poultry in close association with people promote spread of viral and other infectious diseases which have the potential for threatening health, economies and food security around the world. The emergence of new viral diseases/infections, such as Rift Valley fever, West Nile fever, SARS coronavirus, Hendra virus, avian influenza A (H5N1), Nipah virus, Zika virus and swine influenza A (H1N1) virus, from time to time is a glaring example of zoonotic disease threats adversely affecting both animal health and public health, national economies and food and nutrition security globally. Due to a lacuna in the biosecurity, viral diseases like the FMD had reoccurred in countries where these had not been reported for many decades, including the UK, a developed country.

\subsubsection{Biosafety and Biosecurity}

Biosafety and biosecurity are interrelated terms but used in different contexts. The guidelines are developed by WHO, FAO and OIE. Biosafety aims at the protection of person(s) at work and the facilities which are dealing with the biological agents, against their exposure to a disease agent, and prevents unintentional exposure to pathogens/toxins or their accidental release. Thus, biosafety is the application of knowledge, techniques and equipment to prevent personal, laboratory and environmental exposure to potentially infectious agents or biohazards. Biosecurity, unlike biosafety, has divergent meanings in different contexts in which it is used. It deals with the protection of microbiological assets from spill-over, theft, loss, diversion or intentional release from laboratories, preventing the import of certain organisms/ toxins. Biosecurity is a set of preventive measures designed to reduce the risk of intentional transmission of infectious diseases to safeguard the facilities containing sensitive biological materials with the potential of a biological weapon. In brief, biosecurity means bio-risk management. Once a disease is eradicated globally, the policy for keeping the wild and vaccine strains of the virus along with vaccine stocks for emergency use and their subsequent destruction is decided by international agencies like FAO, WHO and OIE based on the recommendations of experts in the area.

\subsubsection{Biosecurity Policies, Protocols and Action Plans}

These include risk assessment; communication and management; quarantine of imported animals at seaports, dry ports and farm; establishment of check posts and vaccination stations at international and interstate borders for clinical surveillance; creation of immune belts at international borders; and planning and conducting 
structured disease surveillance including clinical surveillance and serosurveillance. Biosafety and biosecurity need to be observed at all levels beginning from farm to national and international levels. For handling the most dangerous transboundary disease pathogens, BSL III and BSL IV laboratories are required to ensure biosafety, biosecurity and biocontainment. Proper zoo sanitary measures, such as quarantine; rodent and vector control; disinfection of animal sheds and premises; proper disposal of dung, urine, feed and fodder wastes; and proper carcass disposal, need to be adopted religiously for effective management of EIDs and TADs.

\subsubsection{Biosecurity at International Borders}

Every country needs strict and foolproof biosecurity mechanism at its international borders as a safeguard against the entry of exotic infectious agents/diseases from abroad along with the import of livestock and other animals and their products. For example, India has contiguous and porous borders with countries like Nepal, Bhutan, Pakistan and Bangladesh, besides free trade with Nepal and Bhutan. Since all these countries are vulnerable to TADs, there is a need for regional biosecurity plan to ensure a biosecure region. It would never be possible to have a biosecure country if the bordering countries do not have effective biosecurity in place.

Different countries are at risk for a number of TADs like anthrax, plague, glanders, Lyme disease, contagious equine metritis, Salmonella abortus equi, HPAI virus, FMD virus (SAT 1-3), Lyssavirus, rabies, Hendra and Nipah viruses, West Nile virus, highly pathogenic ND virus, rabbit haemorrhagic disease virus, bovine spongiform encephalopathy (BSE), African horse sickness (AHS), equine encephalomyelitis (EEE, VEE, WEE), equine infectious anaemia, chicken infectious anaemia, equine influenza, vesicular stomatitis, Rift Valley fever, malignant catarrhal fever (MCF) and other TSEs of sheep, goat and deer. Biosecurity measures are required for preventing and containing the ingress of these diseases through international trade. The OIE has facilitated safe trade in animals and animal products by developing effective standards to prevent the spread of animal diseases across the globe. Prevention of transmission of pathogens across intra- and inter-country borders warrants devising of biosecurity measures at par with international standards. Adequate infrastructure comprising check posts and quarantine facilities at seaports, airports and porous international land border are must to check the ingress of viral and other pathogens from across the borders. Diagnostic facilities with trained human resource, and well equipped with instruments and pen-side diagnostic tests/kits, should be in place for ensuring the pathogen-free status of imported livestock and livestock products.

\subsubsection{Biosecurity Measures at National Level}

Biosecurity measures at national level incorporate the components of 'external biosecurity' preventing the ingress of exotic and transboundary animal diseases and 'internal biosecurity' within the country encompassing zonal, compartmental and farm-level biosecurity. Regulations for animal movement through interstate borders 
in India are in place but need strict implementation. Modern detection systems can be used for identification and tracking of animals and animal products to provide information regarding the origin of the animal, and environmental practices used in production and food safety.

\subsubsection{Farm Biosecurity}

For effective disease prevention and control, integration of biosecurity into every operation at the farm is essential. Farm biosecurity should be inclusive of both "bioexclusion' (measures for preventing a pathogen from being introduced to a herd/ flock) and 'biocontainment'. The latter addresses the events after the introduction of the pathogen and its ability to spread among susceptible groups of animals at the farm or further spill-over to other farms.

Strict implementation of biosecurity at farm level has played a crucial role in preventing the spread of diseases. A suitable plan addressing important issues, such as location and layout of the farm, animal health practices in place and general management on the farm, needs to be chalked out. It should be flexible to include new knowledge, concepts and technology. A wide range of biosecurity practices have been recommended for different livestock species and production systems, both for specific infection risks or for disease prevention in general. Biosecurity practices have been recommended for cattle, sheep, pig, poultry and fish production systems.

General biosecurity practices and interventions that can be applicable across species and farms include:

1. Maintaining a closed herd procurement/purchase of animals from known sources

2. Minimising the number of animals purchased/transferred/exchanged and the number of herds from which the animals are introduced

3. Avoiding purchases from markets or dealers

4. Appropriate quarantine and testing of animals upon introduction or reintroduction in farm premises

5. Discouraging farming practices such as hiring a bull or stallion and returning it after the breeding season

6. Avoiding the introduction of biological material of uncertain health status

7. Health and vaccination records should be obtained for all the newly introduced animals

Isolation/quarantine of such animals for 2-8 weeks in a separate quarantine facility should be practiced and the animals during this period should be observed for illness/symptoms and screened for important diseases before mixing with other stock at the farm. Laboratory testing of appropriate samples collected during quarantine against important infectious diseases is recommended. The incoming stock can also be given vaccine against the endemic disease prevalent in the area at least 2 weeks before release from quarantine to boost their protective immunity.

Animal diseases can spread from farm to farm resulting in animal sickness, death and economic losses. Visitors to the livestock farm, disease laboratory, birds, 
rodents, vehicles, feed and fodder and other inanimate objects are often a source of infection. In addition to adverse effects on the economy, there can be negative effects on the environment and human health. The best designs are to implement effective biosecurity practices. Baths by the laboratory workers after and before visiting animal farm or laboratory and putting on gum boots, disposable overall, head gear and gloves should be a mandatory requirement. All effluent from the laboratory should be pre-treated to ensure freedom from pathogens before their disposal to the environment. Disinfectant foot bath for the workers and vehicles entering the farm at the gate, exclusive separate dress and shoes for laboratory and farm workers, minimum movement of the people and animals within the farm during the outbreak period, and personal health and hygiene of the staff are some of the minimum guidelines to strengthen farm biosecurity.

\subsection{Early Disease Reporting and Compensation Play a Key Role in Animal Health Management}

Timely, rapid and accurate disease reporting based on OIE-approved diagnostic tests is a must for effective detection of the pathogen and instituting early response without giving much time for the disease to spread further. To face the new exotic diseases, it is recommended to have a standard SOP in place along with technical guidelines, decision-taking levels along with adequate provision for funds and legal backup. It has been observed that lack or inadequate compensation for culling the diseased and in-contact animals and poultry and negative effect on the sale, sale price and exports deter the farmers from reporting animal diseases in time which eventually leads to the spread of TADs. For example, due to BSE cattle producers in the USA lost over $\$ 1$ billion in exports to Japan. Similarly, HPAI resulted in over \$1 billion loss in poultry exports for Thailand. Trade concerns also discourage the use of preventive vaccinations for some diseases such as HPAI and FMD. Disease-free countries are generally reluctant to import animals or animal products from the countries practicing preventive vaccinations. With these adverse trade considerations, stamping-out policy was adopted by the UK over the vaccination for FMD in 2001 outbreak. From a public health point of view, a vaccination programme might reduce the viral load circulating in a country and thus reduce the risk of HPAI spreading to humans. However, stamping out rather than vaccination is preferred by most of the countries to declare themselves free from disease/infection at the earliest possible to regain access to exports. To ensure the cooperation of livestock farmers, it is essential to provide adequate and timely compensations to reduce the losses suffered by them on account of culling and closing the units for a few months. 


\subsection{Failure in Timely Disease Reporting Hastens Spread of TADs}

The failure of timely disease reporting hastens the spread of TADs within the country as well as between countries. It is believed that the HPAI (H5N1) avian influenza virus might have been circulating in the poultry for months in the affected region before it was reported to the international authorities in 1996 leading to the wide spread of the disease/infection. An ex ante study of the 2001 FMD outbreak of the UK suggested that the FMD virus was probably introduced 3-4 weeks before it was reported and followed by a ban on livestock movements. Earlier reporting and ban on animal movements would have cut the spread of the disease by about $40 \%$.

\subsection{Steps for Control of EIDs and TADs}

It is believed that the EIDs and TADs will continue to remain an ever-growing threat to animal and human health, economic sustenance of the world and global environment well-being. However, it is difficult to predict the number of these diseases which could rapidly escalate in a country or region threatening the animal and human life as well as the economy of that region or nation. The rising global demands for meat, particularly in East and South Asia, have put humans and animals together in numbers never seen before in the world. The fact is that the farm biosecurity in these countries where meat production is growing most rapidly is often poor. This scenario creates a great scope for animal diseases to jump species to create human health problems. Some of these issues were thoroughly described in the IOM Report, 2003, and further debated and discussed by Riemenschneider (2005). The steps suggested include early detection and early response, preparedness plans, decentralisation of government structure, international coordination, understanding of ecology, microbial evolution and viral traffic, expanded surveillance system, disease intelligence, preparedness, collaboration and cooperation among government agencies and cross-field partnerships.

\subsubsection{Preparedness Plans}

In developing countries, the preparedness plans for animal diseases are often unsatisfactory. Incentives such as adequate compensation should be provided to the affected farmers as an impetus for reporting animal diseases. The level of preparedness should be assessed by conducting mock drills. This will help in confidence building for rapid detection and response to both EIDs and TADs that appear suddenly and are capable of spreading to large areas in a short time. Import bans in response to an animal disease outbreak must be based on sound scientific evidence to ensure that the concerned countries also have the incentive to report the disease to international agencies, namely OIE, FAO and WHO. Deficiencies in national 
veterinary services have been attributed for inability in early detection of the disease and response as investigation, and diagnostic services have deteriorated in many regions. A continuing structural upgradation programme for national veterinary services will have to be taken into account for their transformation from providers of services, such as diagnosis, vaccinations and treatment of sick animals to inspection and quality assurance services. Disease surveillance, early warning and emergency preparedness need to be pursued more vigorously in Africa, the Middle East and Southeast Asia as vital components of national veterinary services.

\subsubsection{Decentralisation of Authority}

Though public health and national security are under the perspective of national governments, the decentralised government structure and improved international coordination are essential to address the threat of TADs effectively as they do not respect local, regional or national boundaries. Nevertheless, government support at the administrative level is essential to assure sufficient and timely response to avoid the spread of disease through livestock movement controls, closing of live markets, sharing of diagnostic services, expertise, funding, etc.

\subsubsection{International Coordination}

Technical support and guidance of international agencies, such as FAO, WHO and OIE, are key in the formulation and timely implementation of the plans and modalities for the control and management of EIDs and TADs. The FAO in 1994 established the Global Framework for the control of TADs (GF-TAD) through the Emergency Centre for Transboundary Animal Disease (ECTAD) operations and Emergency Prevention System (EMPRES) for transboundary animal and plant pests and disease initiatives for early warning and response to disease threats, following a collaborative approach to investigation at animal-human-ecosystem interface. These mechanisms have proved to be of immense help and use in the control, prevention and eradication of disease(s).

\subsubsection{Understanding of Ecology, Microbial Evolution and Traffic}

Microbial evolution, particularly viral evolution, is a continuous process. It is, therefore, necessary to conduct basic research on emerging infectious diseases, both viral and another microbial origin, for providing new insights about the factors responsible for the emergence of new microbes. For understanding the ecology of disease, 
social factors, viral and microbial traffic and spread, ecological and demographic changes in human and animal populations due to migration and other factors work in tandem leading to precipitation of emerging infections. These signals for viral and microbial traffic should be seen as warning signs. Biodiversity should include microbes and viruses, and environmental impact assessment should include health aspects into account in development planning.

\subsubsection{Expanded and Robust Surveillance System}

Enhancing surveillance systems by establishing laboratory response network at national, regional and international level is important for which adequate funds should be provided. By linking the laboratories in public and private domains, such networks are expected to enhance the capabilities at all levels to detect and prevent the spread of EIDs, transmitted naturally or intentionally (Anonymous 2003). A network of more than 100 laboratories world over by WHO for a constant survey of influenza viruses is one of the best examples of networking of laboratories for EIDs and TADs. These laboratories should have multidisciplinary teams involving veterinarians, physicians, ecologists, entomologists, vaccinologists, epidemiologists, molecular biologists, immunologists and possibly other specialists.

\subsubsection{Disease Intelligence}

State-of-the art disease surveillance is required having the capability to forecast when and where a particular disease is likely to occur for more targeted surveillance. Such actionable intelligence may derive from the analysis of changes in climatic conditions, vegetation, wildlife demographics, trade pattern or vector demographics and distribution (Anonymous 2003).

\subsubsection{Updating of the Vaccines as per the Current Antigenic Types and Genotypes}

The disease-producing microbes, particularly viruses and bacterial agents, often change their antigenic make-up as a result of spontaneous mutations, and immune pressure when the wild strains of the infectious agent persist in the host in the presence of vaccinal antibodies. RNA viruses having segmented genome are more prone to such antigenic changes as a result of recombination, gene deletion, etc. Influenza A viruses of human and animals continuously evolve new virulent variants by exchanging haemagglutinin $(\mathrm{H})$ and neuraminidase $(\mathrm{N})$ genes of various $\mathrm{H}$ and $\mathrm{N}$ 
types circulating in human, birds, pigs and other species including equines. With the change in antigenic make-up, the current vaccine strains do not provide protection against the new types of the virus. Similar situations occur in FMD virus having seven types and further subtypes, clades and genotypes: PPR virus and Newcastle disease virus, to name a few. New antigenic types of a virus or pathogen may also be introduced from abroad through imported livestock and poultry. Hence, there is a need to have a plan in place to upgrade the vaccine by incorporating the current strains of the pathogen which induce strong and lasting immunity. This will require the setting of repositories of field strains isolated from disease outbreaks, particularly the ones from vaccine failure cases. Such updates of vaccines are routinely followed for influenza vaccines for poultry, equines and human, and FMD and CSF vaccine for livestock.

\subsection{Lessons Learnt from TADs}

\subsubsection{Benefits of Vaccination}

Vaccination is a valuable and well-tested method in preventive veterinary medicine for promoting animal health and welfare and reducing the risk of human exposure to several zoonotic pathogens. Prophylactic immunisation practices, principles and vaccination protocols have helped in significantly reducing the prevalence of many life-threatening viral and bacterial diseases. The risks of not vaccinating their stock on account of lack of awareness among the stakeholders, non-availability of costeffective diagnostics and vaccines, and poor delivery of veterinary services to the livestock farmers can have serious consequences on livelihoods of rural livestock producers. Effective vaccination programmes if implemented properly with a broader perspective are likely to reduce the need for antimicrobials, which in turn can help reduce the risk of emergent antimicrobial resistance. The World Veterinary Association (WVA) and Health for Animals believe that it is essential for the global veterinary profession to educate the public, particularly animal keepers and producers, about the benefits of vaccination for animals and humans.

The major objectives and motive of veterinary vaccines are to protect, improve and promote the health and welfare of companion and food animals; increase the production of livestock in a cost-effective manner; and prevent animal-to-human transmission of infectious diseases from domestic animals and wildlife to humans through animal-origin food, close contact and other mechanisms. These diverse aims have led to different approaches to the development of veterinary vaccines from crude but effective whole-pathogen preparations to molecularly defined subunit vaccines, genetically engineered organisms or chimeras, vectored antigen formulations and naked DNA injections for immunisation of animals. 


\subsubsection{Research on Vaccines and Vaccinology}

The final successful outcome of vaccine research and development is the generation of a product that will be available in the marketplace on demand and suitable to be used in the field to achieve desired outcomes. Successful veterinary vaccines have been produced against major bacterial, viral, protozoan and multicellular pathogens, which led to successful field application and adaptation of novel technologies. These veterinary vaccines have had, and continue to have, a major impact not only on animal health and production but also on human health through increasing safe food supplies, namely milk, meat, eggs and fish, and preventing animal-to-human transmission of infectious diseases. The continued interaction between the researchers from veterinary and medical streams and health professionals will be a major impetus for adapting new technologies, providing animal models of human diseases and confronting new and emerging infectious diseases. Over 100 different veterinary vaccines are currently commercially available (Meeusen et al. 2007).

\subsubsection{Multivalent Vaccines}

Multivalent (bivalent, trivalent and polyvalent) vaccines should be given preference over monovalent vaccines to cover more than one disease prevalent during control programmes to save money, time and other expenses and also to reduce the burden on implementing agencies, such as veterinarians and para-health livestock workers.

\subsubsection{Monitoring of Vaccine Response}

There should be a system in place to conduct post-vaccination sero-monitoring in the field by appropriate agencies for finding evidence for adequate seroconversion in the randomly collected samples as per standard procedure preferably using DIVA tests to differentiate between vaccine-induced immune response and the one induced by the virulent virus.

\subsubsection{Import Risk Analysis}

The application of risk analysis concerning the spread of disease on account of international trade in live animals and their products, namely, import risk analysis (IRA), has been largely driven by the Sanitary and Phytosanitary (SPS) Agreement of the World Trade Organization (WTO). The IRA standard established by the World Organisation for Animal Health (OIE), and associated guidance, meets the needs of the SPS agreement. The use of scenario trees is the core modelling approach adopted to represent the steps necessary for the hazard to occur. There is scope to elaborate scenario trees for commodity IRA so that the quantity of hazard at each step is assessed (Peeler et al. 2015). 
The dependence between exposure and establishment of the hazard suggests that they should fall within the same subcomponent. IRA undertaken for trade reasons must include an assessment of consequences to meet SPS criteria. The integration of epidemiological and economic modelling may open a path for better methods. Matrices have been used in qualitative IRA to combine estimates of entry and exposure, and consequences with likelihood, but this approach has flaws, and better methods are needed. IRA standards and guidance provided by OIE indicate that the volume of trade should be taken into account. Some published qualitative IRAs have assumed current levels and patterns of trade without specifying the volume of trade, which constrains the use of IRA to determine mitigation measures (to reduce risk to an acceptable level) and whether the principle of equivalence, fundamental to the SPS agreement, has been observed. It is questionable whether qualitative IRA can meet all the criteria set out in the SPS agreement. Nevertheless, scope exists to elaborate the current standards and guidance, so that they better serve the principle of science-based decision-making.

\subsection{Disease-Free Zones}

Options for trade from disease-free zones and disease-free compartments and trading in safe commodities are now available to have a positive mechanism for facilitating international trade. In India, FMD-Control Program (FMD-CP) is already in operation intending to create FMD-free zones. Similar zones can be created for other diseases like HS, bluetongue, sheep pox, goat pox, PPR and other important diseases. Compartmental biosecurity is the new concept for the management of biosecurity in a compartment through a single set of biosecurity measures. Creation of zones/compartments will ensure a boost in international trade of livestock and poultry products. In India, legislation regarding the movement of animals across these zones and compartments are required by the central and state governments.

\subsection{Financing Is Key for the Control and Management of TADs}

\subsubsection{Economic Burden from Transboundary Diseases}

The TADs are a threat to animal health and production and cause huge losses to the economy of nations. Recent outbreaks of bovine spongiform encephalopathy (BSE), foot-and-mouth disease (FMD) and highly pathogenic avian influenza (HPAI) have unfolded the real and growing global threat that animal diseases pose to livestock systems and to human health and welfare. The TADs adversely affect the trade in live animals and their products. The detection of one BSE-positive animal in 2003 in 
the USA led to an 80\% drop in beef exports during 2003-2004. Similarly, the losses in the UK were estimated to be over US\$9 billion during the ill-fated 2001 FMD outbreak. The economic losses due to HPAI (H5N1) avian influenza have been estimated from $0.5 \%$ to $1.5 \%$ of GDP in Thailand and Vietnam by Rushton et al. (2005). The 2003 outbreak of avian influenza due to H7N7 strain in the Netherlands destroyed as many as 30 million birds. Direct losses due to FMD in India have been estimated to the extent of INR 230,000 million per annum (Anonymous 2017-18). PPR has been estimated to cause global losses between US $\$ 1.45$ billion and $\$ 2.1$ billion per year. The disease causes economic losses in India to the tune of INR 11,070 million per year (Tripathi et al. 2018).

The FAO of the United Nations and the World Organisation for Animal Health (OIE) have opined that on an average one newly emergent animal disease has been detected per year over the last few decades, while three-fourths of these diseases being zoonotic are transmissible to humans. An outbreak of FMD in the UK in 2001, bluetongue in 2007, equine influenza in Australia in 2007 and India in 2008-2009, and highly pathogenic avian influenza (HPAI) in Maharashtra and adjoining parts of India in 2006 caused severe damage and economic losses to the farmers and industry.

\subsubsection{Availability of Adequate Financial Support}

Availability of adequate financial support for animal health $R \& D$, especially in developing countries, is not always readily ensured. As the livestock keepers in these countries are mostly socio-economically poor, the local and national governments should come forward to support these programmes, particularly for the landless and marginal farmers keeping pigs, sheep, goats, backyard poultry and low-producing bovine stocks by providing incentives or subsidies for diagnostics and vaccines. Raising venture fund for emergency disease control through public and private partnership could be considered to meet the urgent requirements, besides farmer-friendly insurance policies for livestock health protection.

\subsection{3 'One World-One Health' Approach}

For important TADs, such as avian influenza, PPR and FMD, multinational, regional or global programmes under the supervision of FAO, WHO and OIE under 'One Health' concept are suggested for better coordination and results. The 'One WorldOne Health' (OWOH) concept steered by FAO, WHO and OIE has its roots in the interaction between living beings including humans, animals and pathogens, and the environment is considered as a unique dynamic system in which the health of each component is interconnected and dependent with other components. Nowadays, a newly integrated 'One Health One Medicine' approach reflects this interdependence 
with a holistic view of the ecological system. The OWOH can be defined as a collaborative and a multidisciplinary effort at the local, national and global level to guarantee an optimal healthy status for humans, animals and environment. The control of infectious diseases, which have influenced the course of human history, is to be considered strictly related to the One Health concept.

\subsection{Experiences Gained with Some TADS}

\subsubsection{Avian Influenza}

After its first occurrence in 1996 in China, the highly pathogenic avian influenza (HPAI) A virus (H5N1) has affected more than 60 countries in Asia, Europe, Africa and North America. The virus affected wild birds as well as domestic poultry. Sporadic cases of transmission to humans in close contact of infected birds with sizeable mortality raised the pandemic concern of 'bird flu'. After the first report of the H5N1 virus from India and Bangladesh in 2006 and 2007, respectively, both these countries are experiencing outbreaks almost every year. Between FebruaryMarch 2006 and February 2019, India incurred an expenditure of more than INR 1098.7 million, including INR 263.7 million for compensation and INR 835 million on the culling of 8.349 million birds (Anonymous 2018-19). Avian influenza viruses (AIVs) have become a continued threat to global health and economy. After its first outbreak in 2014, the H5N8 HPAI serotype disseminated very fast from Korea to other parts of Asia, Europe and North America, a feature not observed in case of other highly pathogenic AIVs. However, the pathobiological features of the virus that favoured its global translocation are not known. Results of simulation studies undertaken in migratory birds to identify pathobiological features supporting AIV intercontinental dissemination risk suggest that characteristic differences exist among H5N8 and other AIV subtypes, e.g. H5N6 and H5N1 that have not spread as rapidly. Lower infection recovery and mortality rates and migration recovery rates also favour translocation in migratory bird populations. Although India has been reporting H5N1 AIV since 2006, the H5N8 virus was first time reported in 2017 from migratory birds and poultry in the states of Delhi, Madhya Pradesh, Kerala, Karnataka, Punjab and Haryana. Studies undertaken on comparative epidemiology of influenza viruses $\mathrm{H} 5 \mathrm{~N} 1$ and $\mathrm{H} 7 \mathrm{~N} 9$ among human and bird populations to find out similarities and differences between the two viruses in their genetic characteristics, distribution patterns in human and bird populations and postulated mechanisms of global spread (Bui et al. 2014) indicated that H7N9 viruses are diversifying at a much greater rate than H5N1 viruses. Analyses of certain H7N9 strains demonstrated similarities with engineered transmissible $\mathrm{H} 5 \mathrm{~N} 1$ viruses, which make it more adaptable to the human respiratory tract. These differences in the epidemiology of H5N1 and H7N9 viruses in human and birds raise further questions as to how $\mathrm{H} 7 \mathrm{~N} 9$ has spread at a greater rate than the $\mathrm{H} 5 \mathrm{~N} 1$ virus. 


\subsubsection{African Swine Fever}

African swine fever (ASF) is a highly contagious, deadly emerging disease of pigs in many countries. Although first described in 1921 and it affected more than 50 countries in Africa, Europe and South America, several key issues about its pathogenesis, immune evasion and epidemiology remain uncertain (Arias et al. 2017). In the absence of a vaccine, the disease causes greater sanitary, social and economic impacts on swine herds compared to many other swine diseases. Currently, ASF is present in sub-Saharan Africa, Sardinia, the Trans-Caucasus, the Russian Federation and Central and Eastern states of the European Union. The disease continues to spread, with first reports in China (August 2018), Bulgaria (August 2018), Belgium (September 2018) and Vietnam (February 2019) highlighting the increasing threat of ASF to the global pig industry (Netherton et al. 2019). Ongoing outbreaks have also been reported in Hungary, Latvia, Moldova, Poland, Romania, Russia, South Africa, Ukraine, Cambodia, North Korea, Vietnam and Laos. The disease was rampant in China during 2019, and about half of China's breeding pigs died or were slaughtered. The threat of ASF looms large as presently no licensed vaccine is available against this disease, and further research is desired in this area for the development of live attenuated vaccines for ASFV. It has been possible to generate pigs resistant to classical swine fever virus and PRRS virus (Burkard et al. 2018) by using genetic modification of the host species. Genetic modification can be attempted as a viable solution to increase the host resistance to ASFV. Wild suids, namely warthog or bush pig, sequences could be engineered into the domestic pig genome to produce animals in which replication of ASF virus and/or disease burden after ASFV infection is reduced. However, to generate pigs fully resistant to ASFV infection, a more effective strategy such as targeting the virus receptors on the host cell to block the entry of virus and viral replication may be attempted.

Different clinical courses of ASFV infection in pigs have been described based on the virulence of the virus isolates, and sequencing the genomes of isolates of reduced virulence has identified virus genes associated with this phenotype. Targeted gene modifications and deletions and testing of the genetically modified viruses in macrophages and pigs have contributed to an understanding of virulence factors and how the virus modulates host responses. In the absence of a vaccine and rapid spread of ASF in Europe and Asia, the main emphasis should be on strict customs and border protection to keep the negative countries free from ASF virus infection/ disease.

Research is required on priority to explore the virulence genes and genes related to host protection and immune evasion, role of multigene families in antigenic variability, mechanism of evasion of the immune response, factors determining viral persistence and infection outcomes, and interactions between ASFV and wild African suids, which are tolerant to ASFV infection. Such studies will provide a complete understanding of the pathogenesis of ASF. The specific role of different hosts including wild suids, vectors and environmental factors in disease propagation needs to be elucidated for understanding different epidemiological scenarios. In this regard, the northern European scenario in which infected wild boars drive disease 
transmission and maintenance needs to be investigated further. Presently, ASF has become of great significance in China and a real threat to the pig and pork production. The affected countries are planning to compensate for the losses in pork production by increasing broiler poultry production.

Gaps in sanitary control of wild boar populations make ASF control difficult. Raising awareness among veterinarians, hunters and farmers should be the priorities for ASF control. Advances in non-invasive sampling are required to facilitate surveillance in affected areas. Current and future tests need to be optimised for noninvasive matrices. The availability of a confirmatory serological test and cell lines for replacing primary cell cultures should be the priorities for future work. Availability of safe and potent vaccine against ASF could benefit disease control and prevention substantially, but despite some advances such vaccine is still lacking (Arias et al. 2017).

\subsubsection{Foot-and-Mouth Disease}

After the successful eradication of rinderpest from the globe in 2011, foot-andmouth disease (FMD) of cloven-footed animals is another OIE-listed important viral disease inflicting heavy economic losses and adversely affecting the trade of livestock and livestock products from endemic countries to FMD-free nations/ regions. Knight-Jones et al. (2016) have given a detailed account of global FMD research update along with gaps and an overview of global status and research needs. The conclusions are drawn to highlight that currently available vaccines and control tools have enabled FMD eradication from many countries of the developed world. However, in many developing countries, FMD remains uncontrolled. The main reason given is that biosecurity measures that have been fundamental to successful FMD control in the developed country are difficult to be implemented effectively in developing countries due to obvious reasons. In the present scenario, improved vaccines, with longer lasting protection against a wider range of FMDV strains and lower production costs, could be the single most important development to enhance our ability to control FMD. Although encouraging progress has been made with several novel vaccine candidates, addressing key limitations of the current inactivated vaccines, a commercial vaccine is yet awaited. While new discoveries are crucial, current vaccines have been used to effectively control FMD on numerous occasions. However, for imparting better immunity, FMD vaccines should be subjected to adequate quality assurance and be made available in sufficient quantity to provide desired coverage following appropriate strategy. There is also a need for better training and support in the design and execution of vaccine-based FMD control programmes. Another area of research is genetic and molecular studies on the virus to elucidate host-virus interactions. More powerful tools and analyses are increasing our understanding of various aspects of FMDV evolution, ecology and epidemiology. This, in turn, should benefit many areas of FMD research, from basic virology to the vaccine and diagnostic development. Furthermore, improved genetic 
technologies have the potential to reveal information crucial for control, such as transmission chains, vaccine match and level of virus circulation.

FMD control has been prioritised by many governments around the world. Besides traditional bastions of established research institutes in Europe and North and South America, notable work is being conducted in China, India and Africa. Experiences in South America and Europe have shown that through decades of sustained investment FMD can be controlled, even in regions where once it was rampant and control was seemingly impossible. However, if improved and more widespread FMD control is to be achieved, continued investment in FMD research at the local and international level is a must. Improved DIVA diagnostics increase our ability to detect infected animals in vaccinated populations. Greater confidence in the ascertainment of FMD status of animals and products has, in turn, opened the way for international standards for trade and disease control that are more efficient and less restrictive. Rigorous licensing procedures increase the time taken for new technologies for diagnostic kits and vaccines to reach the market. However, if authorisation is less rigorous, substandard products may be released onto the market. Hence, there is a need to balance these two requirements. Relaxation should be provided for necessary changes to the existing technologies, such as changing vaccine strains, particularly when the need is urgent (Knight-Jones et al. 2016).

\subsubsection{Rinderpest: Now a Disease of Past}

Rinderpest, also known as 'cattle plague', was once a deadly serious threat to the livestock industry and agriculture economy in several regions of the globe, particularly in Asia, Africa, Europe and the Americas. It periodically swept through Old World, resulting in devastating epizootics and huge economic losses. The disease could be successfully eliminated from the globe with mass vaccination programmes, zoo sanitary measures, policy support, international cooperation and political will.

The morbidity and mortality rates in newly exposed naïve populations could be as high as 95-100\% leading to enormous economic losses. In India mortality rate of about 200,000 animals were recorded among the affected bovine population of 400,000 per annum during the first half of the 1950s, indicating average mortality of $50 \%$. Throughout the history of humankind, the social, economic and ecological consequences due to rinderpest had been more catastrophic, even changing the history of nations and empires. In India, the presence of rinderpest was confirmed by the Cattle Plague Commission (Hallen et al. 1871). This disease has been conquered successfully by following mass vaccination along with zoo sanitary measures. The FAO declared the global eradication of rinderpest on 28 June 2011, marking it the first ever viral disease of animals eradicated globally about three decades after the eradication of smallpox, a viral disease of humans in 1980 (Yadav et al. 2016). Constraints of availability of quality vaccine in sufficient quality, freeze-drying of vaccines and maintenance of cold chain for a vaccine in tropical countries, lack of infrastructure for structured clinical surveillance and sero-surveillance were some of the limitations in executing the mass vaccination programmes. In India, dividing 
the country into four zones based on the epidemiological picture of the disease and adopting strategic and focused vaccinations at interstate and international borders and migration routes of bovines and caprine for creating immune belts, coupled with rigorous clinical and sero-surveillance, were of great help in achieving freedom from the infection. The financial support and/or technical guidance from FAO, OIE, EU and IAEA were the driving forces in achieving infection-free status for India in the year 2006. With the successful eradication of rinderpest, the livestock sector across the globe became safer, and consequently the living standard of livestock farmers improved.

The success of rinderpest control and eradication proved a rewarding experience and landmark for the veterinary services in India, providing capacity building and confidence among field veterinarians, researchers, policy planners and donor agencies and other stakeholders to undertake a successful control programme of livestock diseases at the national level. The freedom of the country from rinderpest not only enabled the growth of the dairy industry in India but has also boosted the export of meat and other dairy products in the recent decade. Today India tops not only in milk production in the world but is also the largest exporter of buffalo meat. Cost-benefit analyses indicated that every dollar spent on rinderpest control programme gained about $\$ 20$ to the Indian dairy industry through more milk, meat and draft power for better agricultural productivity (Uppal 2011).

\subsection{Conclusion and Prospects}

In the fate of rampant threat due to EIDs and TADs, a diverse, dynamic and wellplanned structured disease surveillance and monitoring approach would be the key for the sustainability and welfare of healthy livestock production systems of any country. Preparedness for combating the prevailing, emerging, re-emerging EIDs and TADs requires robust monitoring and precision detection systems that are flexible, feasible and adaptable under field conditions. In this regard, pen-side diagnostic tests/lab-on-the chip tools are the need of the hour. The hurdles of sampling need to be curtailed opting non-invasive methods for sample collection from different animal species and wildlife. Transparency in disease reporting needs to be adhered to and reported to OIE. Because of trading in animals and animal products, the international obligation for OIE reportable diseases of high importance must be followed by all member countries of WTO. It is high time to apply developed diagnostics and molecular detection tools in the field to ensure fast detection and confirmation of pathogens capable of causing diseases in humans and animals. This must be accompanied by national-level disease surveillance, monitoring and networking to enable an early warning system for infectious diseases based on forecasting (Saminathan et al. 2016). Due priority is also required for development and application of new potent, safe and affordable vaccines and vaccine delivery systems and adopting innovative vaccination programmes and immunomodulatory and effective therapeutic modalities, which would help in devising timely prevention and control strategies against viral and other infectious diseases. Besides these, good manage- 
ment and standard biosecurity and biosafety measures/practices and appropriate hygienic and zoo sanitary and quarantine measures should be observed.

Moreover, on-the-spot control and checking of the spread of pathogens and adequate trade restrictions as envisaged under the SPS agreement of WTO also need to be followed. A holistic vision and approaches are required for timely implementation of these concepts and strategies along with the strengthening of various multidimensional research and development programmes supported by appropriate funding resources. These measures will greatly help to minimise disease incidences and outbreaks, and lessen economic burdens due to infectious animal diseases and boost livestock and poultry health, reproduction and production to strengthen sustainable growth of livestock and poultry industry. Reduction in pandemic threats and public health concerns eventually lead to an improvement in the socioeconomic status and welfare of the society at large under 'One Health' umbrella. Application of artificial intelligence (AI), GPS, remote sensing and traceability in disease detection and management needs priority attention in developing countries. Similarly, the latest techniques of gene editing, base editing, nanotechnologies, electronic nose, etc. should be applied for efficient disease diagnosis and drug delivery. While planning the breeding policies for livestock and poultry, both higher production performance and health of the progeny should be given equal weightage. Modern techniques should be used for developing disease resistance (absolute or partial) in livestock and poultry using indigenous germplasm.

Acknowledgements All the authors of the manuscript thank and acknowledge their respective universities and institutes.

Conflict of interest: There is no conflict of interest.

\section{References}

Anon (2003) Institute of Medicine (IOM) report

Anonymous (2017-18) Annual report, ICAR-Directorate of foot-and-mouth disease, Mukteswar, India, pp $1-88$

Anonymous (2018-19) Annual report, Department of Animal Husbandry, Dairying and Fisheries, Ministry of Agriculture and Farmers Welfare, Govt. of India, New Delhi, pp 61-64. http:// dahd.nic.in

Arias M, Jurado C, Gallardo C, Fernandez-Pinero J, Sanchez-Vizcaino JM (2017) Gaps in African swine fever: analysis and priorities. Transbound Emerg Dis 65(S1):235-247. https://doi. org/10.1111/tbed.12695

Bui C, Bethmont A, Chughtai AA, Gardner L, Sarkar S, Hassan S, Seale H, MacIntyre CR (2014) A systematic review of the comparative epidemiology of avian and human influenza A H5N1 and H7N9-lessons and unanswered questions. Transbound Emerg Dis 63(6):602-620. https:// doi.org/10.1111/tbed.12327

Burkard C, Opriessnig T, Mileham AJ, Stadejek T, Ait-Ali T, Lillico SG et al (2018) Pigs lacking the scavenger receptor cysteine-rich domain 5 of CD163 are resistant to porcine reproductive and respiratory syndrome virus 1 infection. J Virol 92:e00415-18. https://doi.org/10.1128/ JVI.00415-1

Daszak P, Cunningham AA, Hyatt AD (2000) Emerging infectious diseases of wildlife-threats to biodiversity and human health. Science 287:443-449. https://doi.org/10.1111/tbed.12180 
Hallen JHB, Mcleod K, Charles JG, Keer HC, Allijan MM (1871) The cattle plague commission report to government of India. Calcutta Publication, Calcutta, pp 1-999

Knight-Jones TJD, Robinson L, Charleston B, Rodriguez LL, Gay CG, Sumption KJ, Vosloo W (2016) Global foot-and-mouth disease research update and gap analysis: 1—overview of global status and research needs. Transbound Emerg Dis 63(S1):3-13. https://doi.org/10.1111/ tbed. 12528

Lederberg J, Shope RE, Oaks SC Jr (eds) (1992) Emerging infections: microbial threats to health in the United States. National Academy of Sciences, Washington, DC

Malik YS, Dhama K (2015) Zika virus-an imminent risk to the world. J Immunol Immunopathol 17(2):57-59. https://doi.org/10.5958/0973-9149.2015.00019.2

Meeusen ENT, Walker J, Peters A, Pastoret P-P, Jungersen G (2007) Current status of veterinary vaccines. Clin Microbiol Rev 20(3):489-510. https://doi.org/10.1128/CMR.00005-07

Munjal A, Khandia R, Dhama K, Sachan S, Karthik K, Tiwari R, Malik YS, Kumar D, Singh RK, Iqbal HMN, Joshi S (2017) Advances in developing therapies to Combat Zika virus: current knowledge and future perspectives. Front Microbiol 8:2677. https://doi.org/10.3389/ fmicb.2017.01469

Netherton CL, Cornell S, CTO B, Dixon LK (2019) The genetics of life and death: virus-host interactions underpinning resistance to African swine fever, a viral hemorrhagic disease. Front Genet 10:402. https://doi.org/10.3389/fgene.2019.00402

Peeler EL, Reese RA, Thrush MA (2015) Animal disease import risk analysis-a review of current methods and practice: open access article. Transbound Emerg Dis. 62:480-490. https://doi. org/10.1111/tbed.12180

Riemenschneider CH (2005) Avian influenza and other transboundary animal diseases, Director, Liaison Office for North America, Food and Agriculture Organization of the United Nations. Presentation at "Health in Foreign Policy Forum 2005", Washington, DC, 4 Feb 2005

Rushton J, Viscarra R, Guerne Bleich E, McLeod A (2005) Impact of avian influenza outbreaks in the poultry sectors of five South East Asian countries (Cambodia, Indonesia, Lao PDR, Thailand, Viet Nam) outbreak costs, responses and potential long term control. World's Poultry Sci. J. 61(1):491-514

Saminathan M, Rana R, Ramakrishnan MA, Karthik K, Malik YS, Dhama K (2016) Prevalence, diagnosis, management and control of important diseases of ruminants with special reference to Indian scenario. J Exp Biol Agric Sci 4(3S):3338-3367. https://doi.org/10.18006/2016.4 (3s). 338.367

Singh RK, Dhama K, Malik YS, Ramakrishnan MA, Karthik K, Tiwari R, Khandia R, Munjal A, Saminathan M, Sachan S, Desingu PA, Kattoor JJ, Iqbal HMN, Joshi SK (2017) Ebola virus - epidemiology, diagnosis and control: threat to humans, lessons learnt and preparedness plans—an update on its 40 year's journey. Vet Quart 37(1):98-135. https://doi.org/10.1080/01 652176.2017.1309474

Singh RK, Dhama K, Chakraborty S, Tiwari R, Natesan S, Khandia R, Munjal A, Vora KS, Latheef SK, Karthikh K, Malik YS, Singh R, Chaicumpaj W, Mourya DT (2019) Nipah virus: epidemiology, pathology, immunobiology and advances in diagnosis, vaccine designing and control strategies—a comprehensive review. Vet Quart 39(1):26-55. https://doi.org/10.1080/0165217 6.2019 .1580827

Tripathi BN, Kumar N, Barua S (2018) Peste des Petits ruminants: sheep and goat plague. Today and Tomorrow's Printers and Publishers, New Delhi, pp 1-180

Uppal PK (2011) FAO sponsored final project report "National testimonies" under the Global Rinderpest Eradication Programme (GREP)-(GCP/GLO/302/EC), pp 1-134

Yadav MP (2011) FAO sponsored final project report on "Laboratory contributions for rinderpest eradication in India" under the Global Rinderpest Eradication Programme (GREP)-(GCP/ GLO/302/EC), pp 1-58

Yadav MP, Uppal PK, Rao JR (2016) Animal sciences. In: Singh RB (ed) 100 Years of agricultural sciences in India. NAAS, New Delhi, pp 158-258 